UCRL-- 15680

DE86 002604

FINAL TECHNICAL REPORT LLILL SUBCONTRACT 9744809

29 March 1985

William H. Press

Harvard University

Cambridge, Massachusetts 02138

\title{
MSTE
}

March 29, 1985

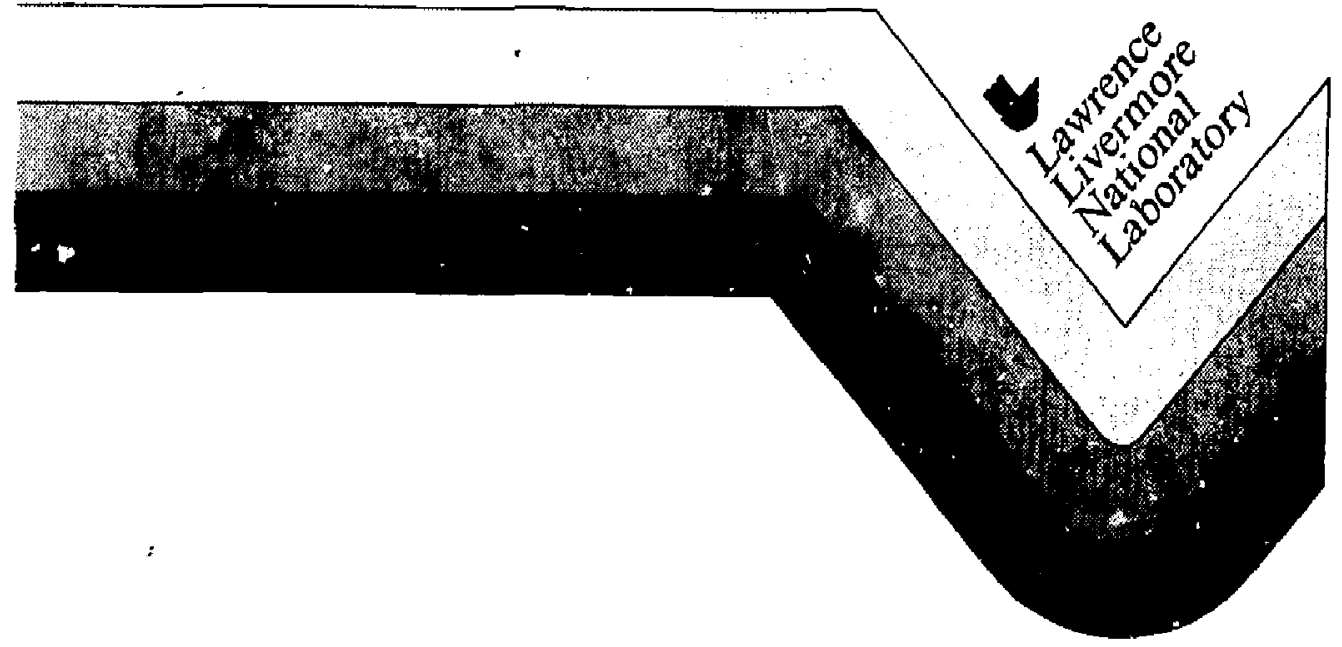




\section{Center for Astrophysics}

60 Garden Street

Cambrldge, Massachusetts 02138
Harvard Collece Observatory

Smithsonlan Astrophysical Observatory

\section{FINAI TECHNICAL REPORT \\ LLL Subcontract 9744809 \\ 29 March, 1985}

Julian H. Krolik (Harvard) and Richard A. London (LLL) investigated the problem of time-steady accretion of gas irradiated by a self-consistently generated quasar-like continuum. A controversy in the leterature was resolved by a careful distinction between the existence of solutions and their stability. Specific criteria were found for when thermal instabilities may disrupt single-phase steady flow. As a function of initial temperature (covering the range from $10^{6} K$ to $10^{8} K$ ), they determined the boundaries of stable flow in the luminosity relative to Eddington $\left(L / L_{E}\right)$-efficiency plane. They then analyzed the thermal and dynamical history of these steady flows. By matching to the observational data that define quasars and type 1 Seyfert galaxies, they found those values of efficiency and $L / L_{E}$ which are required for consistent spherically symmetric steady-flow models: the efficiency must be less than roughly $10^{-2}$, and $L / L_{E}$, between several times $10^{-3}$ and several times $10^{-2}$.

Work by Julian H. Krolik and Timothy R. Kallman established that the observed X-ray sources near the core of the Orion molecular cloud are sufficient to supply all the ionization that is needed to drive the molecular chemistry throughout that portion of the cloud in which the greatest density and diversity of molecular species is found. In small regions within that core, the ionization rate is so high that the molecular balance is significantly different from that in the bulk of the cloud core's volume. They calculated equilibrium models to determine the dependence of molecular abundances on ionization rate, and they constructed an exemplary model to show how this data may be assembled to predict tatal column densities. The observed abundance of certain species, particularly $\mathrm{C}_{2} \mathrm{H}, H \mathrm{C}_{3} \mathrm{~N}$, and $\mathrm{CN}$, unaccounted for in homogeneous models, may be entirely due to the presence of small regions of high ionization rate. It is possible that high abundances of species such as these in other molecular clouds may be used as signals of stellar X-ray emission too faint or too deeply buried in the clouds to be detected by current X-ray telescopes.

Julian H. Krolik and Paul R. Shapiro (University of Texas) put forth a new suggestion for a single-pass, high-gain, $O^{5+}$ ion laboratory laser at $1035 \mathrm{~A}$. A large population inversion was shown to be generated when an $\mathrm{X}$-ray pulse $\mathrm{K}$ shell ionises $\mathrm{O}^{3+}$ ions, followed by Auger ionization to an excited state of $O^{5+}$. Using a strong, pulsed $x$-ray source which is within present capabilities, a gain coefficient of the order $\omega^{\mathrm{cm}}-1$ can be produced in a pure oxygen plasma in coronal ionization equilibrium at $10^{5} \mathrm{~K}$ and at a density of the order of $10^{15} \mathrm{~cm}^{-3}$. Constraints and requirements for the achievement of high gain were analysed. These were shown to 
allow a maximum number of unsaturated gain lengths ranging from several tens to several hundreds, depending upon the size and geometry of the laser configuration.

Noting that the existence of binary stars in globular clusters has a long history of controversy, investigations by Julian H. Krolik found that, at present, the only evidence for binaries in globular ciusters comes from binaries in extreme states, cataclysmic variables and X-ray sources. The existence of the latter is customarily ascribed to tidal capture of red dwarf companions by neutron stars (or possibly solar-mass black holes). In fact, tidal capture should be at least as effective in creating close binaries comprising two main-sequence stars. Subsequent evolution of such binaries is very likely to lead to mass exchange between the two, and possibly merger. In conventional calculations, gravitational radiation drives the binary orbital evolution; recently, it has been shown that in the denser globular clusters, where tidal capture is most effectrive, gravitational encounters with passing field stars also have an important role, both by slow, cumulative compression of the binary orbit, and by occasional catastrophic physical collisions. On the basis of these mechanisms, Krolik predicted that globular clusters should contain substantial numbers of: close binaries; low-mass contact binaries, perhaps similar to the somewhat more massive WUMa stars; and 'blue stragglers', stars lying on the extrapolated main sequence beyond the turn-off. These objects should be most common in the densest regions of the Galaxy's globulat cluster system.

Julian. H. Krolik analyzed the various evolutionary paths possible for highly compact binaries in globular clusters, where they come under the simultaneous influence of gravitational radiation and gravitational encounters with feld stars. The observational appearance of those stages in which mass transfer occurs was discussed, with particular attentioin given to a new class of objects characterized by massive, nonequilibrium accretion disks created by catasstrophic gravitational encounters. Krolik then constructed an analytic model to predict the number of highly compact binaries in each stage of evolution existing at present in the Galaxy's globular cluster system. Both steady transfer binaries, and systems in which a collapsed stellar remnant acquires a massive nonequilibrium accretion disk, were shown to be present in significant numbers. The number of steadily accreting degenerate dwarfs predicted (which can proliably be identified with cataclysmic and weak $\mathrm{X}$ ray sources) was in good agreement with observations $(\sim 1000)$. There should also be $(\sim 1000)$ degenerate dwarfs surrounded by massive disks, whose observational properties are difficult to predict, but are probably quite luminous. Depending on circumstances, they may resemble cataclysmic variables, or possibly red giants. If highly compact binaries can lose angular momentum by no other mechanisms than those considered by Krolik, the number of medium strength $\left(\sim 10^{35}-10^{36}\right.$ ergs s $\left.^{-1}\right)$ X-ray sources produced by steady transfer onto neutron stars $(\sim 10)$ is in conflict with the observations. If the strong $\left(10^{36}-10^{38}\right.$ ergs s $\left.{ }^{-1}\right) \mathrm{X}$-ray sources can be identified with neutron star (or low-mass black hole)-plus-massive-disk systems, their predicted number (several) roughly agrees with the actual one.

J. H. Krolik, A. Meiksin, and P.C. Joss (MIT) calculated the secular evolution of a highly compact binary stellar system, composed of a collapsed object and a 
low-mass secondary star, in the core of a globular cluster. The binary evolves under the combined influences of (i) gravitational radiation losses from the system, (ii) the evolution of the secondary star, (iii) the resultant gradual mass transfer, if any, from the secondary to the collapsed object, and (iv) occasional encounters with passing feld stars. Krolik et al. calculated all these effects in detail, utilizing some simplifying approximations appropriate to low-mass secondaries. The times of encousters with field stars, and the initial parameters specifying those encounters, were chosen by use of a Monte Carlo technique, the subsequent gravitational interactions were calculated utilizing a three-body integrator, and the changes in the binary orbital parameters were thereby determined. They carried out a total of 20 such evolutionary calculations for each of the two cluster core densities ( 1 and $3 x 10^{5}$ stars $\left.p c^{-3}\right)$. Each calculation was continued until the binary was disrupted or until $2 \times 10^{10}$ yr had elapsed.

Julian H. Krolik and J.M. Vrtilek investigated the dynamics of the narrow line regins of Seyfert galaxies. The physical conditions in the vicinity of Seyfert galaxy nuclei that are inferred from line ratio studies and from their X-ray continua suggest that the velocity widths of Seyfert narrow lines are due to entrainment of the emitting clouds in a hot wind flowing out of the center of the galaxy. A dynamical model was constructed to describe this outflow, using a qualitatively realistic gravitational potential for the galaxy and an approximate equation of state for the gas. Line profiles were then derived from this dynamical model and compared to observed profiles. Agreement was quite satisfactory.

Julian H. Krolik and Timothy R. Kallman developed new calculations of the soft X-ray opacity of gas having cosmic elemental abundances, but in a variety of ionization states. They considered coronal ionization equilibrium over the range of temperatures $10^{4}$ to $3 x 10^{8} \mathrm{~K}$ and photoionization equilibrium with power-law and thermal bremsstrahlung spectra over a wide range of ionization parameters. The results are displayed in illustrative figures. For temperatures and ionization parameters commonly found in as .ophysical plasmas, the opacity (particularly to photons of $\leq 1 \mathrm{keV}$ ) can be diminished by substantial factors relative to the state in which all atoms are neutral. As the dominant ionization stages change, the positions and contrasts of prominent $K$-edges also change.

With R. Petre, R.F. Mushotzky, and S.S. Holt, J.H. Krolik presented results of the analysis of 28 Einstein SSS observations of 15 high X-ray luminosity $\left(L_{x}>\right.$ $10^{43.5} \mathrm{etg} \mathrm{s}^{-1}$ ) quasars and Seyfert type I nuclei. The $0.75-4 . \mathrm{keV}$ spectra are in general well fit by a simple model consisting of a power law plus absorption by cold gas. The average spectral index $\langle\alpha\rangle$ is $0.66 \pm .36$, consistent with $\langle\alpha\rangle$ for the spectrum of these objects above $2 \mathrm{keV}$. In all but one case, no evidence was found for intrinsic absorption, with an upper limit of $2 x 10^{21} \mathrm{~cm}^{-2}$. The investigators found no evidence for partial covering of the artive nucleus by dense, cold matter $\left(N_{H}>\right.$ $10^{22} \mathrm{~cm}^{-2}$ ); the average upper limit on the partial covering fraction is 0.5 . There is no obvious correlation between spectral index and $0.75-4.5 \mathrm{keV} \mathrm{X}$-ray luminosity (which ranges from $3 x 10^{43}$ to $10^{47} \mathrm{erg} \mathrm{s}^{-1}$ or with other source properties. The lack of intrinsic X-ray absorption allows the investigators to place constraints on 
the density and temperature of the broad-line emission regions, the narrow line emission segion, and the intergalactic medium.

Krolik reviewed briefly a selection of outstanding questions having to do with star formation. Several suggestions of new areas of work, both theoretical and observational, were developed. Some topics given particular attention were: the most advantageous way to describe the physical states of molecular clouds, the structure of accretion flows onto proto-stellar cores in which angular momentum is significant, and the origin of non-thermal stellar during the approach to the main sequence.

Julian H. Krolik and John C. Raymond outlined the dynamical theory of radiation pressure-driven shocks in winds from early-type stars. After isolating the principal parameters needed for a description of these shocks, they estimated the shocks' principal properties as functions of these parameters. For the special case of time-steady shocks, they then presented detailed numerical calculations of the shocks' internal structure: density, ionization properties. The lack of intrinsic X-ray absorption allows them to place constraints on the density and temperature of the broad-line emission region, the narrow line emission region, and the intergalactic medium.

Respectfully submitted,

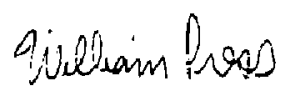

William H. Press

Professor of Astronomy and of Physics

Principal Investigator 